\title{
Caught flat-footed: podocyte damage and the molecular bases of focal glomerulosclerosis
}

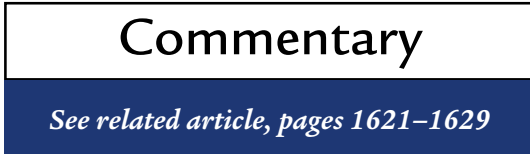

\author{
Dontscho Kerjaschki \\ Department of Pathology, University of Vienna-Allgemeines Krankenhaus, Währinger Gürtel 18-20, A-1090 Vienna, Austria. \\ Phone: 43-1-40400-5176; Fax: 43-1-40400-5193; E-mail: dontscho.kerjaschki@akh-wien.ac.at.
}

J. Clin. Invest. 108:1583-1587 (2001). DOI:10.1172/JCI200114629.

The renal glomerulus, the site of plasma ultrafiltration and the production of primary urine (1), is the locus of a number of progressive disorders that lead to chronic renal insufficiency. Before the advent of renal replacement therapies, these diseases invariably led to death by uremia. Still, while these treatments save, or at least extend, patient's lives, they do so at an enormous price, both in human and financial terms.

\section{The podocyte and focal glomerulosclerosis}

The prevention of chronic renal insufficiency would require therapies that specifically interfere with the pathogenesis of the various underlying glomerular diseases. Disappointingly however, the molecular mechanisms involved lie in uncharted territory. These conditions can result from varied causes: systemic metabolic disorders, such as diabetes; autoimmune complex formation, as occurs in membranous and lupus nephropathy; or primary podocyte diseases, such as steroid-sensitive minimal change nephrosis, or the steroid-insensitive condition focal segmental glomerulosclerosis (FSGS).

Other than diabetes, FSGS now represents the leading cause of renal insufficiency, both in the general patient population and among those whose disease recurs following transplantation. The basis for the increased prevalence of FSGS in recent years is as mysterious as its pathogenesis, and even the best therapies for this disorder are strictly empirical. However, the recent emergence of the visceral glomerular epithelial cell, or podocyte, as the culprit in this and several other glomerular diseases raises new hope for developing rational treatments.

Podocytes owe their name to their elegant, complex shape that is con- served in vertebrates from zebrafish to humans. Like an octopus, the podocyte's cell body emits thick extensions. These structures branch to form the foot processes that cover the surface of the glomerular capillary loops like the interdigitating fingers of two hands (Figure 1). The foot processes adhere to the ECM proteins of the glomerular basement membrane (GBM) - mainly laminin, the proteoglycans agrin and perlecan, type IV collagen - via the basal cell membrane (the "sole" of the podocyte, see Figure 2 , red area). Foot processes are joined laterally by slit diaphragms (SD), which presumably reinforce the structure against tensile force resulting from filtration pressures of about 40 $\mathrm{mm} \mathrm{Hg}$. SD may also provide an isoporous filter and thus contribute to the size limit of the glomerular filter. Alternatively, SD may impede filtrate flow, thus increasing the hydraulic pressure within the inflated GBM and preventing its collapse. The lateral membrane domains of foot processes into which the slit diaphragms insert are designated in the following as slit diaphragm domain (see Figure 2, blue area). Finally, there is the major proportion of the foot processes cell membrane that extends into the urinary space, also called the apical domain (see Figure 2, green area).

\section{Glomerular diseases result in a pathological increase of glomerular porosity and proteinuria}

Podocytes react in an apparently stereotypic pattern, retracting their foot processes into their cell bodies to form a flattened, simple epithelium - thus recapitulating in reverse their embryonic development. Whether this is a general cause or a consequence of glomerular filter damage is uncertain, but at least for FSGS and minimal change nephrosis (2), podocyte damage appears to be the primary cause of disease. However, the molecular basis of podocyte stability and, conversely, podocyte flattening, were long unknown.

\section{Molecular architecture of the podocyte foot processes}

Several approaches, some systematic and others serendipitous, have recently yielded an unprecedented wealth of information on functionally significant podocyte membrane and cytoskeletal proteins. In addition, the availability of cultured immortalized murine podocytes that retain several critical phenotypic properties of podocytes in situ were a definite stimulus and a valuable experimental tool (3). Together with older data, the recent findings provide the basis of a provisional and almost certainly incomplete blueprint of the foot processes (Figure 3).

\section{The slit diaphragm domain: a crowded place}

The discovery of several novel proteins and the characterization of their physical and functional interactions

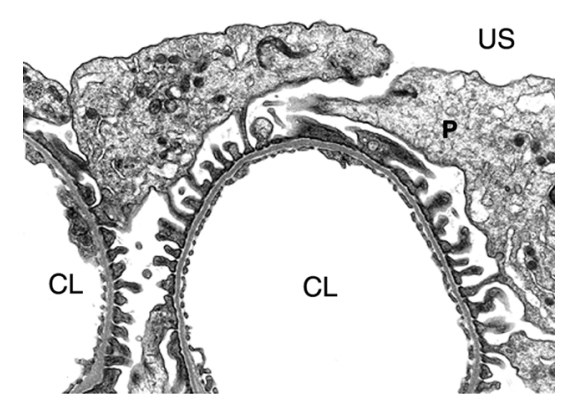

Figure 1

Survey view of a rat glomerular capillary loop. Cell bodies of podocytes $(P)$ extend into the urinary space, while their foot processes attach in a regular, interdigitating pattern. US, urinary space; $C L$, capillary lumen. $\times 12,000$. 


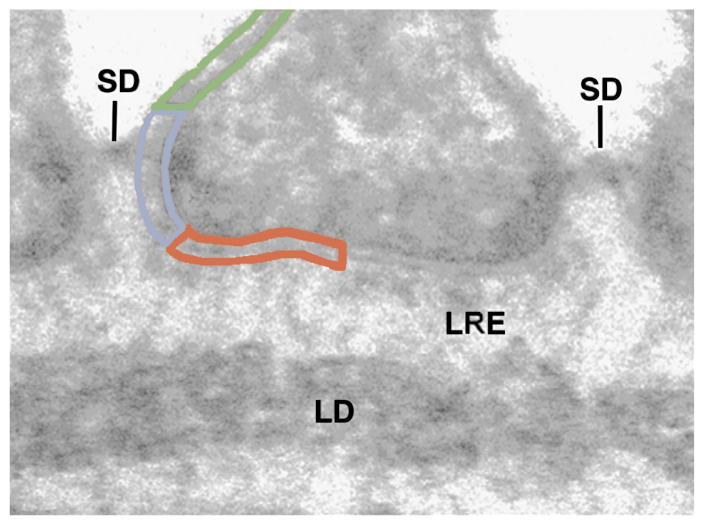

\section{Figure 2}

High power view of the basal half of a podocyte attached to the lamina rara externs (LRE) of the glomerular basement membrane. The slit diaphragms (SD) insert laterally into the podocyte cell membrane. Three membrane domains are marked: green indicates the apical cell membrane above the slit diaphragm; blue marks the membrane of the slit diaphragm domain; red indicates the cell membrane at the base, or "sole" of the foot process. LD: lamina densa. $\times 140,000$.

helped define the region of the insertional membrane of the SD as a major locus of podocyte function. A breakthrough that has renewed interest in glomerular biology and pathology was the discovery of a membrane protein called nephrin as a major component of the SD complex (reviewed in ref. 4). Mutations of the nephrin gene NPHS1 were identified by positional cloning as the pathogenic cause of familial Finnish nephropathy, in which a severe nephrotic syndrome is evident even in utero and is accompanied by the complete flattening of foot processes. Similarly, homozygous knock-out mice generated by targeted inactivation of the Nephrin gene fail to develop foot processes and are nephrotic (5). Intravenous injection of a monoclonal anti-nephrin antibody likewise leads to proteinuria (6). Taken together, these results indicate that nephrin is crucial for the development of the slit diaphragm and for the maintenance of the correct permeability of the glomerular capillary filter.

Nephrin is a 1241 amino acid protein with a large extracellular domain containing eight C2-type IgG like domains, a single membrane spanning domain, and a cytoplasmic portion that contains potential phosphorylation sites. The putative geometry of nephrin initially suggested that two molecules aligned in an antiparallel fashion could constitute the entire slit diaphragm. However, experiments in several laboratories failed to show dimerization of purified recombinant nephrin, suggesting that additional components are required to assemble the slit diaphragm. Recent evidence indicates that nephrin is a component of lipid rafts, which also contain a podocyte specific 9-O-acetylated GD3 ganglioside. Intravenous injection of a monoclonal antibody to this ganglioside results in incomplete flattening of foot processes, dislocation, and tyrosine phosphorylation of nephrin (7).

Recently, gene trapping by retrovirusmediated mutagenesis has identified a podocyte protein related to nephrin that has been termed NEPH-1 (8). The similarities between these two proteins include numbers of IgG-like domains and phenotypes in homozygous knock out mice. It is not yet clear how this protein interacts and cooperates with nephrin. Another success of positional cloning was the discovery of the stomatin-like protein podocin (9). Mutations of this protein have been found in a family with hereditary FSGS. As with stomatin in erythrocyte membranes, podocin forms a hairpin structure with both termini in the cytoplasm. It also forms aggregates and organizes lipid rafts, as Schwarz et al. (10) report in this issue of the JCI.

In addition to these characteristic podocyte proteins, the SD contains several other, more widely distributed components. The CD2-associated protein CD2AP, for example, was originally defined as a protein that binds the cytoplasmic portion of the T-lymphocyte and natural killer cell surface marker CD2. CD2AP possesses three SH3 domains and a coiled coil domain, which serve as attachment sites for other proteins. The significance of $\mathrm{CD} 2 \mathrm{AP}$ for podocyte biology was discovered by chance in CD2AP-knock out mice that unexpectedly developed a severe nephrotic syndrome and died around week 7 post partum (11). Podocytes of these animals were flattened and resembled kidney cells in FSGS patients. P-cadherin, another one of the SD domain transmembrane proteins, co-localizes with the zonula occludens associated protein $\mathrm{ZO}-1$, a member of the membrane associated guanylate kinase (MAGUK) family that also binds to F-actin (12). Intracellularly, $\mathrm{P}$-cadherin is associated with the signalling proteins $\alpha$-, $\beta$-, and $\gamma$-catenin. The relation of $\mathrm{P}$-cadherin to nephrin and NEPH-1 is not clear. Likewise, the large cadherin homologue FAT has been found in the slit diaphragm domain, but its relation to the other proteins located there remains to be determined (13).

A complex of nephrin, podocin and $\mathrm{CD} 2 \mathrm{AP}$ is emerging as the functional unit upon which the SD assembles. These proteins are tightly associated and are embedded into lipid rafts, as shown in the article by Schwarz et al (10). Podocin may be critical for the stability of this complex by forming aggregates and lipid rafts. Intriguingly, its binding dramatically activates the signalling capabilities of nephrin (14). The scaffolding proteins $\mathrm{ZO} 1$ and CD2AP form links with F-actin and may trap other yet unidentified proteins by virtue of their $\mathrm{SH} 3$ and coiled coil domains. It also remains to be elucidated which cooperative roles NEPH-1, P-cadherin, FAT, and nephrin play in the formation and stability of slit diaphragms.

\section{The sticky sole of the foot}

The basal membrane domain of foot processes expectedly contains several adhesion proteins that link podocytes to the extracellular matrix. Recently it has become clear that the proteins encountered at this domain also form specialized, interconnected complexes. The integrin $\alpha_{3} \beta_{1}$, localized more than ten years ago to the soles of foot processes (15), is essential for maturation of podocytes, as shown by the loss of foot processes development in $\alpha_{3}$-deficient mice (16). Nevertheless, $\alpha_{3} \beta_{1}$ is normally localized in FSGS and other glomerular diseases in which podocytes are extensively flattened, 
suggesting that $\alpha_{3} \beta_{1}$ integrin constitutes stable, static bonds between podocytes and the GBM. Podocyte $\alpha_{3} \beta_{1}$ associates on its cytoplasmic side with paxillin, talin, and vinculin (17), which mediate its connection to the actin cytoskeleton. An integrin linked kinase monitors and influences the state of activity of the $\alpha_{3} \beta_{1}$ integrin and serves signalling (18).

Also found at the sole of the podocyte's foot is the dystoglycan (DG) complex, originally defined as an adhesion system of skeletal muscle cells. $\alpha$ - and $\beta$-DG occur at very high density at the soles of foot processes, as seen by immunoelecton microscopy in humans and rats $(19,20)$. DG binding to the Helix pomatia lectin apparently accounts for the ability of that agent to stain the podocyte foot, as was first reported three decades ago (21). Several other non-muscular and epithelial cells have recently been shown to express DG-complexes, and while each has a characteristic composition, the core structure invariably contains the two non-covalently bound subunits $\alpha$ - and $\beta$-DG. $\alpha$-DG contains a cluster of sialic acid-rich carbohydrate side chains that bind electrostatically to cationic regions of ECM proteins such as laminin and agrin. $\beta$-DG spans the cell membrane and binds to cell typespecific actin linker proteins (reviewed in ref. 22). In podocytes, utrophin takes the place of the muscle protein dystrophin, and there may be more, yet undiscovered isoforms and homologues. Podocytes also contain syntrophin-1, which attaches to $\beta$-DG and may bind ion channels or other membrane proteins.

The function of the DG-complex at the soles of foot process is uncertain. However, based on findings in myocytes, the glomerular DG-complex could provide an actin-directed positioning system by which podocytes actively control the exact spacing of matrix proteins - and thus the porosity and permeability of the GBM. The proteinuria and the flattened morphology of foot process that occur when DG expression is diminished in experimental and human diseases could support this view. Alternatively, the DG-complex may serve as a regional organizer for membrane protein clusters in the sole of foot processes, perhaps interacting with the $\alpha-\delta$ sarcoglycans, which surround $\beta$-DG. $\delta$-Sarcoglycan links DG to $\beta 1$-integrins in other cell types (23), and we have demonstrated a sarcoglycan-integrin linkage in podocytes as well (our unpublished data). PDZ-protein binding motifs on $\beta$-DG could link the DG-complex to membrane protein clusters via as yet unidentified scaffolding proteins.

Recently, the PDZ-protein MAGI-1 was found to associate with megalin (24), a polyspecific receptor of the LDL-receptor family. Megalin was previously identified as pathogenic anti-

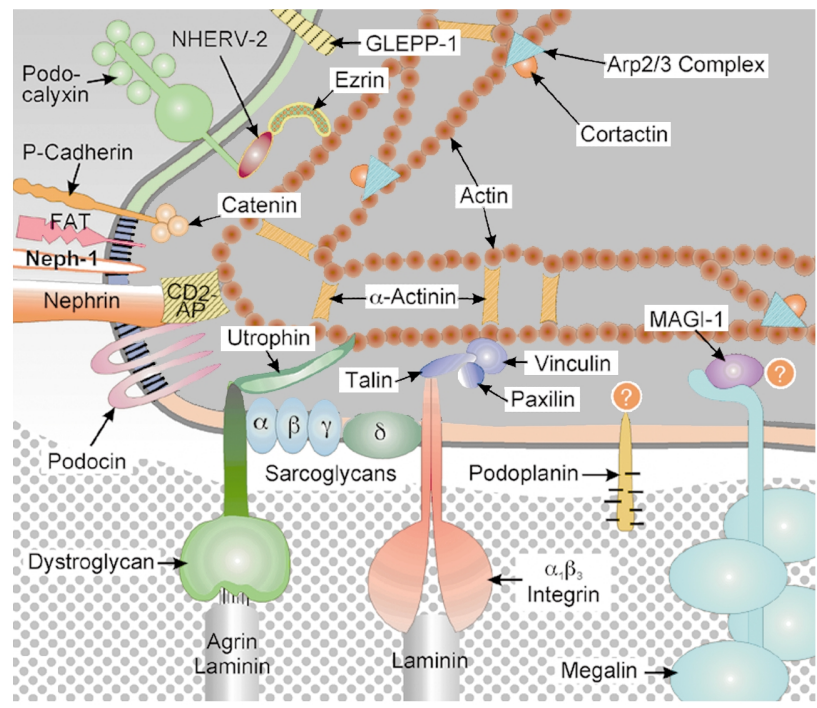

\section{Figure 3}

Schematic drawing of a latero-basal portion of a podocyte, similar to the area marked in Figure 2. In this greatly simplified graph, molecules are not drawn to a correct scale or shape. The three membrane domains defined in the preceding figure are marked here with the same color code.

gen of Heymann nephritis and localized to clathrin coated pits all over the surface of podocytes, and particularly also to the soles of foot processes (25). In podocytes, megalin serves as endocytic receptor for lipoproteins (26). The intracellular association of megalin with MAGI-1 could provide an additional link for protein complexes at the soles of foot processes.

\section{The apical cell membrane domain}

The apical membrane of foot processes forms yet another functional unit, one in which the actin cytoskeleton is indirectly associated with the CD34 related membrane glycoprotein podocalyxin $(27,28)$. The membrane above the SD is endowed with a negatively charged surface coat that is primarily made up of podocalyxin, and the absence or chemical modification of this protein causes collapse of this entire domain.

Expression of recombinant podocalyxin was recently shown to confer antiadhesive properties to MDCK cells, presumably also reflecting the protein's crucial structural role. This property depends on its highly negative charge that is provided by about 20 sialic acid residues per molecule, as well as several sulfate groups. Recently, however, it was discovered that podocalyxin also contributes directly to the stability of foot processes, because a genetic knock out resulted in immature glomeruli with flattened embryonic podocytes (29). This may be due in part to the lack of negative charge on the cell surface, but it may also be caused by the connection of podocalyxin to the actin cytoskeleton. The PDZ protein NHERV-2, which was originally discovered as a regulator of ion channels, mediates this interaction. NHERV-2 associates with ezrin, which in turn attaches to the actin cytoskeleton (30). Phosphorylation events presumably regulate these interactions, and it is therefore possible that the membrane phosphatase GLEPP 1, located in the same membrane domain, is involved in this machinery. Whatever the precise function of GLEPP 1, it can be apparently compensated by other proteins, as a genetic knock out shows only subtle effects on the anatomy of podocytes (31).

\section{Actin, the central organizer}

As seen in Figure 3, F-actin localizes to the submembranous regions of all three 
membrane domains of podocytes (17). Apparently, the membrane at the foot processes' soles is attached to this actin meshwork via dystoglycan and utrophin or via integrin, talin, paxillin, and vinculin. In the SD area, attachment of the nephrin/podocin complex is mediated by CD2AP. P-cadherin may use ZO-1 for this purpose. Finally, in the apical membrane domain NHERV-2 and ezrin indirectly link podocalyxin to actin.

Actin fiber formation is a highly dynamic process that is governed by continuous assembly and disruption of filaments. Active growth of actin filaments requires special molecular assembly devices, such as the $\operatorname{Arp} 2 / 3$ complex, which attaches to an actin filament and nucleates the growth of a new branching filament (32). The Arp $2 / 3$ complex is activated by many proteins, including cortactin, which was recently localized to the edges of podocyte extensions in vitro (33), indicating active actin polymerization. In vivo, cortactin is localized to the entire submembranous cytoskeleton in normal foot processes, but it becomes undetectable in flattened foot processes (our unpublished data). This suggests that the dynamics of the submembranous actin meshwork is profoundly changed in flattened foot processes.

These findings assign a central role to the submembranous actin cytoskeleton for maintaining the infrastructure of the membrane domains and for the stability of the podocytes shape. This critical role is highlighted by the finding that a genetic knock out of the actin bundling protein $\alpha$-actinin 4 causes podocyte damage resembling that of the silenced genes for nephrin, NEPH-1, CD2AP, integrin $\alpha_{3}$-chain, or podocalyxin. Hence, an intact submembranous actin cytoskeleton is apparently indispensable for maintaining the podocyte architecture.

\section{Toward a molecular pathogenesis of flat feet}

The hereditary human glomerular diseases mentioned above have already contributed substantially to our understanding of the basic processes of foot process damage, but from a clinical point of view, "idiopathic" cases are far more common. Because damage affects more than a single protein, the basis of disease in these cases is more complex and difficult to define. Even in "simple" experimental model diseases the number of molecules affected may be extremely large. An instructive example is the SFGF-like disease seen in homozygous Mpv17-knock out mice (34), which leads to heavy proteinuria and complete podocyte flattening. It appears that overproduction and extracellular release of oxygen radical species (ROS) cause the podocyte damage, as this phenotype can be completely rescued by oxygen radical scavengers (35). ROS degrade matrix proteins (36), and may dramatically affect the binding activity of adhesion proteins (Kojima and Kerjaschki, unpublished observations), but nothing is known about the cooperation of ROS with proteases in glomeruli, or their effect on the membrane proteins of podocytes. Is it possible that ROS afflicted damage to some of the critical podocyte proteins, such as nephrin or podocalyxin, compromise their function and thus induce foot process flattening? Could indiscriminate damage of podocyte membrane proteins funnel into a common pathway of podocyte reaction, e.g. by destabilizing the submembranous actin cytoskeleton?

\section{Synopsis and perspective}

The podocyte surface membrane is divided into three membrane domains with different locations, protein compositions, and functions. Each of the domains contains a core of clustered proteins, some of which are critical for maintaining not only the integrity of their home domain, but also for the stability of the global podocyte architecture. One important example, related to the clustering of the slit diaphragm associated proteins nephrin, CD2AP, and podocin, is provided by Schwarz et al. in this issue (10).

A common infrastructural link of all domains of foot process and podocyte membranes is provided by the submembranous actin cytoskeleton. However, we do not know how podocytes receive signals from their environment and instruct their functional units to react, because our knowledge of podocyte surface receptors is still rudimentary. It would not be surprising, if for example chemokine receptors, such as CCR13 and CCR14 (37), and/or receptors for growth factors, such as for the basic fibroblast growth factor (38), play a major role in maintaining or destroying the podocytes' stability.
At the molecular level, FSGS and presumably also minimal change nephrosis are not definite disease entities, but rather syndromes, as isolated or combined damage to any of the listed podocyte proteins could result in identical clinical and histopathological features. It is not clear yet at which molecular target therapeutic interventions would best be directed, but the unraveling of the composition, functional hierarchy, and cooperation of podocyte domains is definitely a good start.

\section{Acknowledgments}

This work was supported by the Austrian Fonds zur Förderung der Wissenschaftlichen Forschung, SFB 05, Projekt 07, and by the European Community, Grant EU-GLG1-CZ2000-00619.

1. Brenner, B.M., Baylis, C., and Deen, W.M. 1976. Transport of molecules across renal glomerular capillaries. Physiol. Rev. 56:502-517.

2. Farquhar, M.G., Vernier, R.L., and Good, R.A 1957. An electron microscopic study of the glomerulus in nephrosis, glomerulonephritis and lupus erythematosus. J. Exp. Med. 106:649-660.

3. Mundel, P., Reiser, J., and Kriz, W. 1997. Induction of differentiation in cultured rat and human podocytes. J. Am. Soc. Nephrol. 8:697-705.

4. Tryggvason, K.J. 1999. Unraveling the mechanisms of glomerular ultrafiltration: nephrin, a key component of the slit diaphragm. J. Am. Soc. Nephrol. 10:2440-2555.

5. Putaala, H., Soininen, R., Kilpelainen, P., Wartiovaara, J., and Tryggvason, K. 2001. The murine nephrin gene is specifically expressed in kidney, brain and pancreas: inactivation of the gene leads to massive proteinuria and neonatal death. Hum. Mol. Genet. 10:1-8

6. Topham, P.S., et al. 1999. Nephritogenic mAb 51-6 is directed at the extracellular domain of rat nephrin. J. Clin. Invest. 104:1559-1566.

7. Simons, M., et al. 2001. Involvement of lipid rafts in nephrin phophorylation and organization of the glomerular slit diaphragm. Am. J. Pathol. 159:1069-1077.

8. Donoviel, D.B., et al. 2001. Proteinuria and perinatal lethality in mice lacking NEPH1, a novel protein with homology to NEPHRIN. Mol. Cell. Biol. 21:4829-4836.

9. Boute, N., et al. 2000. NPHS2, encoding the glomerular protein podocin, is mutated in autosomal recessive steroid-resistant nephrotic syndrome. Nat. Genet. 24:349-354.

10. Schwarz, K.S., et al. 2001. Podocin, a raft-associated component of the glomerular slit diaphragm, interacts with CD2AP and nephrin. J. Clin. Invest. 108:1621-1629.

11. Shih, N.Y., et al. 1999. Congenital nephrotic syndrome in mice lacking CD2-associated protein. Science. 286:312-315.

12. Reiser, J., Kriz, W., Kretzler, M., and Mundel, P. 2000. The glomerular slit diaphragm is a modi fied adherens junction. J. Am. Soc. Nephrol. 11:1-8.

13. Inoue, T., et al. 2001. FAT is a component of glomerular slit diaphragms. Kidney Int. 59:1003-1012.

14. Huber, T.B., Kottgen, M., Schilling, B., Walz, G., and Benzing, T. 2001. Interaction with podocin facilitates nephrin signaling. J. Biol. Chem. 276:41543-41546.

15. Kerjaschki, D., et al. 1989. A beta 1-integrin recep- 
tor for fibronectin in human kidney glomeruli. Am. J. Pathol. 134:481-489.

16. Kreidberg, J.A., et al. 1996. Alpha 3 beta 1 integrin has a crucial role in kidney and lung organogenesis. Development. 122:3537-3547.

17. Drenckhahn, D., and Franke, R.P. 1988. Ultrastructural organization of contractile and cytoskeletal proteins in glomerular podocytes of chicken, rat, and man. Lab. Invest. 59:673-682.

18. Kretzler, M., et al. 2001. Integrin-linked kinase as a candidate downstream effector in proteinuria. FASEB J. 15:1843-1845.

19. Regele, H.M., et al. 2000. Glomerular expression of dystroglycans is reduced in minimal change nephrosis but not in focal segmental glomerulosclerosis. J. Am. Soc. Nephrol. 11:403-412.

20. Raats, C.J., et al. 2000. Expression of agrin, dystroglycan, and utrophin in normal renal tissue and in experimental glomerulopathies. Am. J. Pathol. 156:1749-1765.

21. Brown, D., et al. 1986. Regional heterogeneity of glycoconjugate distribution in the glomerulus revealed by lectin-gold cytochemistry and SDSPAGE. Am. J. Pathol. 125:601-610.

22. Henry, M.D., and Campbell, K.P. 1999. Dystroglycan inside and out. Curr. Opin. Cell. Biol. 11:602-607.

23. Yoshida, T., Pan, Y., Hanada, H., Iwata, Y., and Shigekawa, M. 1998. Bidirectional signaling between sarcoglycans and the integrin adhesion system in cultured L6 myocytes. J. Biol. Chem. 273:1583-1590.

24. Patrie, K.M., Drescher, A.J., Goyal, M., Wiggins,
R.C., and Margolis, B. 2001. The membrane-associated guanylate kinase protein MAGI-1 binds megalin and is present in glomerular podocytes. J. Am. Soc. Nephrol. 12:667-677.

25. Kerjaschki, D., and Farquhar, M.G. 1983 Immunocytochemical localization of the Heymann nephritis antigen (GP330) in glomerular epithelial cells of normal Lewis rats. J. Exp. Med. 157:667-686.

26. Kerjaschki, D., et al. 1997. Pathogenic antibodies inhibit the binding of apolipoproteins to megalin/gp330 in passive Heymann nephritis. J. Clin. Invest. 100:2303-2309.

27. Kerjaschki, D., Sharkey, D.J., and Farquhar, M.G. 1984. Identification and characterization of podocalyxin-the major sialoprotein of the renal glomerular epithelial cell. J. Cell Biol. 98:1591-1596.

28. Kershaw, D.B., et al. 1997 Molecular cloning and characterization of human podocalyxin-like protein. Orthologous relationship to rabbit PCLP1 and rat podocalyxin. J. Biol. Chem. 272:15708-15714.

29. Doyonnas, R., et al. 2001. Anuria, omphalocele and perinatal lethality in mice lacking the CD34 related protein podocalyxin. J. Exp. Med. 194:13-27.

30. Takeda, T., McQuistan, T., Orlando, R.A., and Farquhar, M.G. 2001. Loss of glomerular foot processes is associated with uncoupling of podocalyxin from the actin cytoskeleton. J. Clin. Invest. 108:289-301.

31. Wharram, B.L., et al. 2000. Altered podocyte structure in GLEPP1 (Ptpro)-deficient mice associated with hypertension and low glomerular fil tration rate. J. Clin. Invest. 106:1281-1290.

32. Volkmann, N., et al. 2001. Structure of Arp2/3 complex in its activated state and in actin filament branch junctions. Science. 293:2456-2459.

33. Welsch, T., Endlich, N., Kriz, W., and Endlich, K 2001. CD2AP and p130Cas localize to different F-actin structures in podocytes. Am. J. Physiol. Renal. Physiol. 281:F769-F777.

34. Weiher, H., Noda, T., Gray, D.A., Sharpe, A.H., and Jaenisch, R. 1990. Transgenic mouse model of kidney disease: insertional inactivation of ubiquitously expressed gene leads to nephrotic syndrome. Cell. 62:425-434.

35. Binder, C.J., Weiher, H., Exner, M., and Kerjaschki, D. 1999. Glomerular overproduction of oxygen radicals in Mpv17 gene-inactivated mice causes podocyte foot process flattening and proteinuria: a model of steroid-resistant nephrosis sensitive to radical scavenger therapy. Am. J. Pathol. 154:1067-1075.

36. Riedle, B., and Kerjaschki, D. 1997. Reactive oxygen species cause direct damage of EngelbrethHolm-Swarm matrix. Am. J. Pathol. 151:215-231.

37. Van Den Berg, J.G., et al. 2000. Interleukin-4 and interleukin-13 act on glomerular visceral epithelial cells. J. Am. Soc. Nephrol. 11:413-422.

38. Floege, J., et al. 1995. Basic fibroblast growth factor augments podocyte injury and induces glomerulosclerosis in rats with experimental membranous nephropathy. J. Clin. Invest. 96:2809-2819. 